\title{
Gravitational radiation from the magnetic field of a strongly magnetized star
}

\author{
R. Lapiedra ${ }^{1}$ and J. A. Miralles ${ }^{2}$ \\ 1 Departament d'Astronomia i Astrofísica, Universitat de València, Campus de Burjassot, 46100 Burjassot (València), Spain \\ 2 Departament de Física Aplicada, Universitat d'Alacant, Apartat de Correus 99, 03080 Alacant, Spain
}

Received 21 November 2002 / Accepted 5 March 2003

\begin{abstract}
We consider the electromagnetic (e.m.) field of a compact strongly magnetized star. The star is idealized as a perfect conducting sphere, rigidly rotating in a vacuum, with a magnetic moment not aligned with its rotation axis. Then we use the exterior e.m. solution, obtained by Deutsch (1955) in his classic paper, to calculate the gravitational waves emitted by the e.m. field when its wavelength is much longer than the radius of the star. In some astrophysical situations, this gravitational radiation can overcome the quadrupole one emitted by the matter of the star, and, for some magnetars, would be detectable in the near future, once the present detectors, planned or under construction, become operative.
\end{abstract}

Key words. gravitational waves - stars: magnetic fields - stars: neutron

\section{Introduction}

Large magnetic fields are supposed to be present in many compact stars. These fields can induce a distortion in the shape of the star, breaking the axial symmetry around the rotation axis if this axis is not aligned with the magnetic moment (Bocquet et al. 1995). Based on this, Bonazzola \& Gourgoulhon (1996) have computed the gravitational wave emission of a distorted neutron star when the magnetic moment is not aligned with the rotation axis of the star. They claim that the deformation may be large enough to lead to a detectable signal by VIRGO. In a similar line, Heyl (2000) has considered the case of magnetized white dwarfs. He concludes that LISA may be able to detect the gravitational waves from the two fastest rotating stars out of a given sample of potential white dwarf sources of gravitational radiation. On the other hand, Palomba (2001) has considered the gravitational radiation emitted from young magnetars. He concludes that the corresponding signals may be detected by the advanced interferometers of LIGO-II and LIGO-III for a magnetic field $B>10^{15} \mathrm{G}$ and moderate values of the distortion parameter, $\beta$.

In this paper we calculate the continuous gravitational radiation due to the e.m. field itself, of a compact strongly magnetized star. We compare this result with the quadrupole gravitational radiation of the distorted mass of the star, whatever the origin of this distortion may be: due to the action of the magnetic field of the star or not. It is shown that for some magnetars, the gravitational radiation due to the e.m. field can be of the same order of the quadrupole gravitational radiation of the

Send offprint requests to: R. Lapiedra, e-mail: ramon. lapiedra@uv.es mass, or even grater. However, this is no longer true for the list of distorted magnetized white dwarfs considered by Heyl in the above cited paper. We also find that a hypothetical magnetar in our galaxy, with $\sim 10^{15} \mathrm{G}$, would be within the expected limit of detection for continuous gravitational radiation.

It should be noted that the e.m. field of the star, as a source of gravitational waves, is not a bounded source, which means that to calculate the distant gravitational wave emitted by this source, we cannot use the standard quadrupole approximation.

\section{Calculation of the gravitational waves emitted by the e.m. field of a magnetized star}

If $T_{\alpha \beta}$ is the energy-momentum tensor of an e.m. field, $F_{\alpha \beta}$, in vacuum, the linearized Einstein equations can be written, using a harmonic coordinate system as follows

$\eta^{\alpha \beta} \partial_{\alpha} \partial_{\beta} h_{\gamma \delta}=-16 \pi G T_{\gamma \delta}$

where $h_{\alpha \beta}$ is the metric perturbation, $\eta_{\alpha \beta}$ denotes the Minkowski metric and

$T_{\alpha \beta}=\frac{1}{4 \pi}\left(\eta^{\gamma \delta} F_{\alpha \gamma} F_{\beta \delta}-\frac{1}{4} F^{\gamma \delta} F_{\gamma \delta} \eta_{\alpha \beta}\right)$.

Here, since we are dealing with an e.m. field in vacuum, i.e., the electric four-current is zero, the corresponding energymomentum tensor is conserved, that is, $\partial_{\alpha} T^{\alpha \beta}=0$. On the other hand, $T^{\alpha \beta}$ is traceless. Then, $h^{\alpha \beta}$, is also traceless and $\partial_{\alpha} h^{\alpha \beta}=0$.

The star is idealized as a perfect conducting sphere, rigidly rotating in a vacuum, with a magnetic moment that is not aligned with its rotation axis. For the e.m. field we will use the 
exterior solution obtained by Deutsch (1955) in the Minkowski space-time ${ }^{1}$. Recently, Rezzolla et al. (2000) have considered general relativistic effects on the solution and have obtained the e.m. field solution for a background metric of a slowly rotating star. However, in the present paper we are working in an idealized scenario in order to outline the general features of the gravitational radiation due to the e.m. field of the star, therefore the original Deutsch solution will suffice, without any general relativistic correction. It can be seen that this correction for the gravitational wave $h$ is of the order of $\left(R_{\mathrm{Sch}} / R\right) h$, where $R_{\mathrm{Sch}}$ is the Schwarzschild radius of the star. So, given that in our case $R_{\mathrm{Sch}} / R \sim 1 / 3$, we see that the calculations presented here are only a crude estimation of the gravitational radiation emitted by the e.m. field of the star.

Let $R$ be the radius of the compact rotating star, which is $\sim 10 \mathrm{~km}$ in the case of a pulsar, and let $\omega$ be its angular velocity. Now, even in the less favorable case of a millisecond pulsar, we will have $\omega R \ll 1$. So, we can expand the exterior e.m. solution of Deutsch in powers of $\omega R$, and truncate it at the lowest order in $\omega R$. To begin with, we consider the particular case where $\chi$, the angle between the magnetic moment and the rotation axis of the star, is about $\pi / 2$. This value of $\pi / 2$ is one of the peaks of the histogram of $\chi$ values for different pulsars (Rankin 1990). Using values of $\chi$ near $\pi / 2$ allows us to neglect those terms proportional to $\cos \chi$ for the moment. Later on, we will consider the general case, i.e., where $\chi$ is arbitrary. The approximate magnetic and electric fields components, in the Gaussian system of units, are the following,

$$
\begin{aligned}
B_{r}= & \frac{2 \mu}{r^{3}}(1+\omega r) \mathrm{e}^{i \kappa} \sin \theta \sin \chi \\
B_{\theta}= & \frac{\mu}{r^{3}}\left(-1-i \omega r+\omega^{2} r^{2}\right) \mathrm{e}^{i \kappa} \cos \theta \sin \chi, \\
B_{\phi}= & \frac{i \mu}{r^{3}}\left(-1-i \omega r+\omega^{2} r^{2}\right) \mathrm{e}^{i \kappa} \sin \chi \\
E_{r}= & \frac{\omega \mu R^{2}}{2 r^{4}}\left(-3-i \omega r+\omega^{2} r^{2}\right) \mathrm{e}^{i \kappa} \sin 2 \theta \sin \chi, \\
E_{\theta}= & \omega^{3} \mu\left[\frac{\omega^{2} R^{2}}{6}\left(\frac{6}{\omega^{4} r^{4}}-\frac{6 i}{\omega^{3} r^{3}}-\frac{3}{\omega^{2} r^{2}}+\frac{i}{\omega r}\right)\right. \\
& \left.\times \cos 2 \theta+\frac{i}{\omega r}-\frac{1}{\omega^{2} r^{2}}\right] \mathrm{e}^{i \kappa} \sin \chi, \\
E_{\phi}= & \frac{i \omega \mu}{r^{2}}\left[\frac{R^{2}}{6 r^{2}}\left(6-6 i \omega r-3 \omega^{2} r^{2}+i \omega^{3} r^{3}\right)-1+i \omega r\right] \\
& \times \mathrm{e}^{i \kappa} \cos \theta \sin \chi .
\end{aligned}
$$

${ }^{1}$ There is a misprint in the equation for the $\theta$ component of the electric field in Deutsch's paper. The correct expression is

$$
\begin{aligned}
E_{\theta}= & \frac{\omega \mu}{R^{2}}\left\{-\frac{R^{4}}{r^{4}} \sin 2 \theta \cos \chi+\mathrm{e}^{i(\phi-\omega t)} \sin \chi\right. \\
& \left.\times\left[\left(\frac{\rho}{\rho h_{2}^{\prime}+h_{2}}\right)_{\rho=\omega R} \quad \frac{\rho h_{2}^{\prime}+h_{2}}{\rho} \cos 2 \theta-\frac{h_{1}}{h_{1}(\omega R)}\right]\right\} .
\end{aligned}
$$

Here, it means that we take the real part of the complex expressions, and the components of the electric and magnetic fields are related to the spherical coordinates, $(r, \theta, \phi)$, of the inertial system of reference which is located at the center of the star. We take $\theta$ as the angle between the rotation axis of the star and the direction we are considering. Finally, $\kappa \equiv \phi+\omega(r-t)$, and $\mu$ is the magnetic dipole moment of the star. All the components above are calculated up to order $O(\omega R)$.

In order to calculate $h_{\alpha \beta}$, we use the retarded solution of Eq. (1), which can be written for Cartesian coordinates as follows

$h_{\alpha \beta}=4 G \int \frac{\mathrm{d}^{3} x^{\prime}}{\left|\boldsymbol{x}^{\prime}-\boldsymbol{x}\right|} T_{\alpha \beta}\left(t-\left|\boldsymbol{x}^{\prime}-\boldsymbol{x}\right|, \boldsymbol{x}^{\prime}\right)$.

We emphasize again that, since the domain of $T_{\alpha \beta}$ is not bounded, we cannot apply the standard quadrupole approximation and so we must calculate this integral directly.

To calculate the integral in Eq. (9) first we have to obtain the Cartesian components of fields $\boldsymbol{B}$ and $\boldsymbol{E}$. For the Cartesian components of the magnetic field we have

$B_{1}=\sin \theta \cos \phi B_{r}+\cos \theta \cos \phi B_{\theta}-\sin \phi B_{\phi}$,

$B_{2}=\sin \theta \sin \phi B_{r}+\cos \theta \sin \phi B_{\theta}+\cos \phi B_{\phi}$,

$B_{3}=\cos \theta B_{r}-\sin \theta B_{\theta}$,

and a similar expression for the electric field components.

Since we will calculate $h_{\alpha \beta}$ to the lowest significant order in $\omega R$, i.e., order zero, we see from Eqs. (3)-(8) that, leaving aside the terms which go as $1 / r^{2}$ and $1 / r$, the components of the electric field, $\boldsymbol{E}$, are at least $\omega R$ times lower than the components of the magnetic field, $\boldsymbol{B}$. Next we will see that the contribution to the gravitational waves of those terms in $\boldsymbol{B}$ and $\boldsymbol{E}$ going as $1 / r^{2}$ and $1 / r$ are negligible when compared to the main contribution due to the other terms in the e.m. field given in Eqs. (3)-(8). This means that the electric field can be neglected in the calculation of the integral (9) and only the Cartesian components of $\boldsymbol{B}$ have to be considered.

Taking into account Eqs. (3)-(5), after an elementary calculation, we find

$$
\begin{aligned}
B_{1}= & \frac{\mu}{r^{3}}\left[\left(3 \sin ^{2} \theta-1\right) \cos \phi \cos \kappa-\sin \phi \sin \kappa\right] \sin \chi \\
& +O\left(1 / r^{2}, 1 / r\right),
\end{aligned}
$$

$$
\begin{aligned}
B_{2}= & \frac{\mu}{r^{3}}\left[\left(3 \sin ^{2} \theta-1\right) \sin \phi \cos \kappa+\cos \phi \sin \kappa\right] \sin \chi \\
& +O\left(1 / r^{2}, 1 / r\right),
\end{aligned}
$$

$B_{3}=\frac{3 \mu}{2 r^{3}} \sin 2 \theta \cos \kappa \sin \chi+O\left(1 / r^{2}, 1 / r\right)$,

where $O\left(1 / r^{2}, 1 / r\right)$ stands for the remaining terms which go as $1 / r^{2}$ and $1 / r$.

This magnetic field produces an energy-momentum tensor, $T_{\alpha \beta}$, which depends on $t$ only through $\sin \kappa$ and $\cos \kappa$. But to achieve our proposal of calculating the gravitational radiation of the e.m. of the star, we have to obtain the time derivative of $h_{\alpha \beta}$, and therefore we only need the time dependent part 
of $h_{\alpha \beta}$, which is denoted by $P\left(h_{\alpha \beta}\right)$. So, in a similar notation, what we need is simply $P\left(T_{\alpha \beta}\right)$. Then, from Eqs. (13)-(15), after some elementary algebra, we can see that $P\left(T_{\alpha \beta}\right)$ depends on $t$ exclusively through $\cos 2 \kappa$. So, let us write

$P\left(T_{\alpha \beta}\right) \equiv S_{\alpha \beta} \mathfrak{R}\left[\mathrm{e}^{2 i \kappa}\right]$,

where $\mathfrak{R}[\cdots]$ stands for "real part" and $S_{\alpha \beta}$ stands for the corresponding real factor which does not depend on $t$. Then, the time retarded value of $P\left(T_{\alpha \beta}\right)$ will take the expression,

$P\left(T_{\alpha \beta}\left(t-\left|\boldsymbol{x}^{\prime}-\boldsymbol{x}\right|, \boldsymbol{x}^{\prime}\right)\right)=S_{\alpha \beta} \mathfrak{R}\left[\mathrm{e}^{2 i \kappa} \mathrm{e}^{2 i \omega\left|\boldsymbol{x}^{\prime}-\boldsymbol{x}\right|}\right]$.

To begin with the calculation of $P\left(h_{\alpha \beta}\right)$, let us consider first the component $P\left(h_{12}\right)$. According to Eq. (2), and bearing in mind that we can neglect the electric field, we have

$T_{12}=-\frac{B_{1} B_{2}}{4 \pi}$.

Then, from Eqs. (13) and (14), we easily obtain

$$
\begin{aligned}
B_{1} B_{2}= & \frac{\mu^{2}}{2 r^{6}}\left[\left(3 \sin ^{2} \theta-1\right)^{2} \sin 2 \phi \cos ^{2} \kappa-\sin 2 \phi \sin ^{2} \kappa\right. \\
& \left.+\left(3 \sin ^{2} \theta-1\right) \cos 2 \phi \sin 2 \kappa\right] \sin ^{2} \chi+O\left(1 / r^{n}\right),
\end{aligned}
$$

where $O\left(1 / r^{n}\right)$ stands for the remaining terms which go as $1 / r^{n}$, with $n$ any positive integer number less than 6 .

Now, from Eq. (19), let us calculate the time dependent part $P\left(T_{12}\right)$. After some elementary algebra we get

$$
\begin{aligned}
P\left(T_{12}\right)= & \frac{\mu^{2}}{8 \pi r^{6}}\left[i \mathrm { e } ^ { 2 i \omega ( r - t ) } \left(\frac{9}{4} \mathrm{e}^{4 i \phi} \sin ^{4} \theta-\frac{9}{4} \sin ^{4} \theta\right.\right. \\
& \left.\left.+3 \sin ^{2} \theta-1\right) \sin ^{2} \chi\right]+O\left(1 / r^{n}\right) \equiv S_{12} \mathfrak{R}\left[\mathrm{e}^{2 i \kappa}\right] .
\end{aligned}
$$

Here, as in Eq. (19), we do not specify the terms $O\left(1 / r^{n}\right)$, since, as reported above and as it will be made clear at the end of the present calculation of $P\left(h_{12}\right)$, the contribution of these terms to $P\left(h_{12}\right)$ are negligible when compared with the main contribution due to terms explicitly present in Eq. (20), which goes as $1 / r^{6}$.

So, according to Eqs. (9) and (17), we have for $P\left(h_{12}\right)$,

$P\left(h_{12}\right)=4 G \int \mathrm{d}^{3} x^{\prime} \mathfrak{R}\left[\frac{\mathrm{e}^{2 i \omega\left|x^{\prime}-\boldsymbol{x}\right|}}{\left|\boldsymbol{x}^{\prime}-\boldsymbol{x}\right|} S_{12} \mathrm{e}^{2 i \kappa}\right]$.

Then, let us expand the expression $\mathrm{e}^{i \omega\left|\boldsymbol{x}^{\prime}-\boldsymbol{x}\right|} /\left|\boldsymbol{x}^{\prime}-\boldsymbol{x}\right|$ in spherical harmonics, $Y_{L}^{M}(\theta, \phi)$, according to the well known formula

$$
\begin{aligned}
\frac{\mathrm{e}^{2 i \omega\left|\boldsymbol{x}^{\prime}-\boldsymbol{x}\right|}}{\left|\boldsymbol{x}^{\prime}-\boldsymbol{x}\right|}= & 8 \pi i \omega \sum_{L=0}^{\infty} j_{L}\left(2 \omega r_{<}\right) h_{L}^{(1)}\left(2 \omega r_{>}\right) \\
& \times \sum_{M=-L}^{L} Y_{L}^{M *}(\theta, \phi) Y_{L}^{M}\left(\theta^{\prime}, \phi^{\prime}\right),
\end{aligned}
$$

where $j_{L}$, and $h_{L}^{(1)}$ are the spherical Bessel functions of first class, and the spherical Hankel functions, respectively, and where $r_{<}\left(r_{>}\right)$is the smaller (larger) of $|\boldsymbol{x}|$ and $\left|\boldsymbol{x}^{\prime}\right|$.
Let us substitute Eq. (22) in the integrand of Eq. (21). Here, when integrating on the radial variable $r^{\prime}$, we will have to calculate the following integrals

$$
I_{L} \equiv \int_{R}^{\infty} \frac{j_{L}\left(2 \omega r_{<}\right) h_{L}^{(1)}\left(2 \omega r_{>}\right) \mathrm{e}^{2 i \omega r^{\prime}}}{r^{\prime 4}} \mathrm{~d} r^{\prime} .
$$

Now, we are going to see that the different integrals $I_{L}$ go like $(\omega R)^{L}$, and so, to zero order in $(\omega R)$, the only integral $I_{L}$ to be retained is $I_{0}$. To demonstrate this, notice that we can write

$I_{L}=h_{L}^{(1)}(2 \omega r) I_{L 1}+j_{L}(2 \omega r) I_{L 2}$

where

$I_{L 1}=\int_{R}^{r} \frac{j_{L}\left(2 \omega r^{\prime}\right) \mathrm{e}^{2 i \omega r^{\prime}}}{r^{\prime 4}} \mathrm{~d} r^{\prime}$

$I_{L 2}=\int_{r}^{\infty} \frac{h_{L}^{(1)}\left(2 \omega r^{\prime}\right) \mathrm{e}^{2 i \omega r^{\prime}}}{r^{4}} \mathrm{~d} r^{\prime}$.

Here, $r$ means the radial coordinate of the observation point.

On the other hand, it can be seen that

$h_{L}^{(1)}(2 \omega r \gg 1) \approx(-i)^{(L+1)} \frac{\mathrm{e}^{2 i \omega r}}{2 \omega r}$,

$j_{L}(2 \omega r \gg 1) \approx \frac{\cos \left[2 \omega r-\frac{(L+1) \pi}{2}\right]}{2 \omega r}$.

In Appendix A we show that $I_{L 1}$ goes like $\alpha(\omega R)^{L}+$ $\beta(\omega R)^{3} \ln (\omega R)$. So, when considering the different integrals $I_{L 1}$, the dominant integral is $I_{01}$. Looking at any convenient table of definite integrals one gets

$I_{01}=\frac{1}{3 R^{3}}+O(\omega R)$.

Now let us come to the integrals $I_{L 2}$. Using Eq. (27) and integrating by parts it is easy to conclude that

$I_{L 2} \propto \frac{\mathrm{e}^{4 i \omega r}}{\omega^{2} r^{5}}$

and we see that $I_{L 2}$ is completely negligible as compared to $I_{L 1}{ }^{2}$.

Therefore, to zero order in $\omega R$, the only significant integral $I_{L}$ is $I_{0}$. According to Eqs. (23)-(30), its value is

$I_{0} \approx-i \frac{\mathrm{e}^{2 i \omega r}}{6 \omega r R^{3}}+O(\omega R)$

All this means that, when substituting Eq. (22) in Eq. (21), the only values of $L$ and $M$ which must be retained are $L=0$, and $M=0$. So, for the integral in Eq. (21), we have the simpler expression

$$
\begin{aligned}
P\left(h_{12}\right)= & -\mathfrak{R}\left[4 G \omega \mu^{2} \sin ^{2} \chi \mathrm{e}^{-2 i \omega t} \int \frac{j_{0}\left(2 \omega r_{<}\right) h_{0}^{(1)}\left(2 \omega r_{>}\right)}{r^{6}}\right. \\
& \left.\times \mathrm{e}^{2 i \omega r^{\prime}} Y_{0}^{0^{*}}(\theta, \phi) Y_{0}^{0}\left(\theta^{\prime}, \phi^{\prime}\right) f\left(\theta^{\prime}\right) \mathrm{d}^{3} x^{\prime}\right]
\end{aligned}
$$

2 This result becomes apparent from Eq. (23) if we take the observer at an infinite distance from the very beginning. 
where $f\left(\theta^{\prime}\right) \equiv-\frac{9}{4} \sin ^{4} \theta^{\prime}+3 \sin ^{2} \theta^{\prime}-1$, and we have used the fact that the integration over $2 \pi$ of $\mathrm{e}^{4 i \phi}$ is zero. Finally, since $Y_{0}^{0}=1 / \sqrt{4} \pi$, and using Eqs. (23) and (31), we obtain

$P\left(h_{12}\right) \simeq \frac{2 G \mu^{2} \sin ^{2} \chi}{15 R^{3} r} \sin 2 \omega(r-t)+O(R \omega)$.

Now, we will argue why the above result is correct in spite of the fact that, in its calculation, we have considered only the terms in $\boldsymbol{B}$ which go as $1 / r^{3}$, neglecting the ones going as $1 / r^{2}$, and $1 / r$. To begin with, one could easily think that these omitted terms in $\boldsymbol{B}$ would contribute to $h_{\alpha \beta}$ through some integrals such as $I_{L}$, in Eq. (23), with the denominator $r^{\prime 4}$ now substituted by $r^{\prime 3}, r^{\prime 2}, r^{\prime}$, or 1 . Then, following the same strategy to calculate these new integrals as the one we followed above to calculate $I_{L}$, one can see that the new integrals are, at least, $\omega R$ times smaller than $I_{0}$. So, to order zero in $\omega R$, Eq. (33) gives the correct expression for $P\left(h_{12}\right)$, assuming, as we have done, that $\chi \sim \pi / 2$.

Once we have calculated $h_{12}$, let us calculate $h_{11}$ always assuming that $\chi \sim \pi / 2$. To begin with, according to Eq. (2), we write for $T_{11}$ (bear in mind that, in our approximation in $\omega R$, we can take the electric field as zero)

$T_{11}=\frac{1}{8 \pi}\left(B_{3}^{2}+B_{2}^{2}-B_{1}^{2}\right)$.

From Eqs. (13)-(15), we find after some trivial calculation

$$
\begin{aligned}
& P\left(T_{11}\right)=\frac{\mu^{2}}{8 \pi r^{6}} \mathrm{e}^{2 i \omega(r-t)} \sin ^{2} \chi \\
& \quad \times\left[\frac{9}{2} \mathrm{e}^{4 i \phi}\left(\sin ^{2} \theta-\frac{3}{2} \sin ^{4} \theta\right)-\frac{9}{4} \sin ^{4} \theta+3 \sin ^{2} \theta-1\right] .
\end{aligned}
$$

So, we must do the integral

$P\left(h_{11}\right)=\frac{G}{2 \pi} \int \mathrm{d}^{3} x^{\prime} \mathfrak{R}\left[\frac{\mathrm{e}^{2 i \omega\left(\left|x^{\prime}-x\right|\right)}}{\left|\boldsymbol{x}^{\prime}-\boldsymbol{x}\right|} S_{11} \mathrm{e}^{2 i \kappa}\right]$,

where, according to Eq. (16), $S_{11}$ is implicitly defined by $P\left(T_{11}\right) \equiv S_{11} \mathfrak{R}\left[\mathrm{e}^{2 i k}\right]$.

From here on, we must do the same kind of approximate calculation that we have done in the preceding case of $h_{12}$, again only retaining the values of $L=0$ and $M=0$. The corresponding final result is

$P\left(h_{11}\right) \simeq-\frac{2 G \mu^{2} \sin ^{2} \chi}{15 R^{3} r} \cos 2 \omega(r-t)+O(\omega R)$,

which can be compared with expression (33) for $h_{12}$.

A similar calculation gives zero for the double time component, $h_{00}$, and also for the remaining space components $h_{i j}$, to order zero in $\omega R$, except for $h_{22}$, and, of course, $h_{21}$. As long as the three mixed components $h_{0 i}$ are considered, they are zero, since $T_{0 i}$ is also zero, attending that it is a linear combination of the components of the electric field, which has been taken as equal to zero in the present approximation. Then, since $h_{\alpha \beta}$ is traceless, we will have $h_{22}=-h_{11}$. So we have completed the calculation of the components of $h_{\alpha \beta}$, for $\chi \approx \pi / 2$, in a TT gauge.

Although the above expressions for the gravitational wave $h_{\alpha \beta}$ have been obtained by assuming $\chi \approx \pi / 2$, it is easy to show that, to order zero in $\omega R$, these expressions are also valid for any value of $\chi$, since the energy-momentum tensor verifies

$$
P\left(T_{\alpha \beta}\right)=P\left(T_{\alpha \beta}\right)\left(\sin ^{2} \chi\right)+P\left(T_{\alpha \beta}\right)(\sin 2 \chi),
$$

and the second term on the right hand side gives no contribution to the integral of Eq. (9).

Then, Eqs. (33) and (37), together with $h_{21}=h_{12}$, and $h_{22}=$ $-h_{11}$, give us the nonzero components of the gravitational wave produced by the exterior e.m. field of our star, for any value of $\chi$, to zero order in $\omega R$.

Notice that we have mentioned not just the e.m. of the star, but its "exterior" e.m. field. So, what about the contribution of the interior e.m. field of the star to $h_{\alpha \beta}$ ? To answer this question, one must consider the fact that this interior field is coupled to the electric currents present inside the star, or at least on its surface. So, as opposed to the exterior of the star, now, in the interior, the divergence of the e.m. energy-momentum tensor will not be zero. Therefore we need to incorporate the contribution of the currents to the overall interior energy-momentum tensor of the e.m. field plus matter. But this overall energymomentum tensor is, in any case, a bounded source of gravitational waves, and a bounded source whose dimension, $R$, is much smaller than the wavelength, $\omega^{-1}$, of the emitted gravitational waves. Then, to calculate the contribution of this interior overall energy-momentum tensor to $h_{\alpha \beta}$, we can use the well known quadrupole approximation (see any text on General Relativity: for example, Landau \& Lifshitz 1971):

$h_{i j} \approx \frac{2 G}{r} \frac{\partial^{2}}{\partial t^{2}} \int T_{00}\left(t-r, x^{\prime}\right) x_{i}^{\prime} x_{j}^{\prime} \mathrm{d}^{3} x^{\prime}$,

where $T_{00}$ refers to this overall interior tensor e.m. and $h_{i j}$ to the corresponding contribution to the total gravitational wave.

A rough estimation of this integral is trivially

$h \sim \frac{G \omega^{2} \mu^{2}}{R r}$,

where $h$ stands for the amplitude of any of the space components of $h_{i j}$ above, and $\mu$ for the internal magnetic dipole moment of the star. This value of $h$ must be compared with the corresponding estimation from Eqs. (33) and (37), i.e.,

$h \sim \frac{G \mu^{2}}{R^{3} r}$.

If in Eqs. (40) and (41) we consider a similar value for $\mu$, we see that the interior $h$ is $(\omega R)^{2}$ times smaller than the external one. This means that, in our approximation, the contribution of the interior magnetic field of the star to the gravitational waves, emitted by the overall e.m. field of the star, can be neglected. Then, definitely, the gravitational wave emitted by the e.m. of our star to zero order in $\omega R$ is given by Eqs. (33) and (37), which can be written, after bringing back the speed of light, $c$, as follows,

$$
\begin{aligned}
& P\left(h_{12}\right)=\frac{2 G \mu^{2} \sin ^{2} \chi}{15 c^{4} R^{3} r} \sin 2 \omega(r-t), \\
& P\left(h_{11}\right)=-\frac{2 G \mu^{2} \sin ^{2} \chi}{15 c^{4} R^{3} r} \cos 2 \omega(r-t) .
\end{aligned}
$$

All the other components are zero, except, of course, $P\left(h_{21}\right)=$ $P\left(h_{12}\right)$, and $P\left(h_{22}\right)=-P\left(h_{11}\right)$. 


\section{Discussion}

In the last section we have calculated the gravitational waves emitted by the e.m. field of a compact strongly magnetized star in a very simplified scenario using the e.m. solution of Deutsch, mainly because we have ignored the distortions that the ionized atmosphere of the star can introduce in the e.m. field. Nevertheless, our calculation has made it clear that in such a star, a significant amount of gravitational radiation could be present even when the equatorial eccentricity of the star is completely negligible. If this eccentricity is not negligible, and in order to compare both types of gravitational radiation, i.e., that due to the matter of the star and the other due to its e.m. field, let us consider the energy-momentum pseudo-tensor, $t^{i j}$, of the gravitational field, as found in Landau \& Lifshitz (1971) for example. Then, let us calculate $t^{0 i}$ for our gravitational wave. Bearing in mind Eq. (42), and after a straightforward calculation we find:

$t^{0 i}=\frac{c^{3}}{32 \pi G} \partial_{t} h_{a b} \partial_{t} h_{a b} n_{i}$,

where index $a$ and $b$ take values 1,2 , and $n_{i}$ stands for the 3 -unit vector in the observation direction. More explicitly:

$t^{0 i}=\frac{c^{3}}{16 \pi G}\left[\left(\partial_{t} h_{12}\right)^{2}+\left(\partial_{t} h_{11}\right)^{2}\right] n_{i}$,

that is to say

$t^{0 i}=\frac{c^{3} \omega^{2}}{4 \pi G}\left[P\left(h_{12}\right)^{2}+P\left(h_{11}\right)^{2}\right] n_{i}$.

So, we find for the radiated power, $P$,

$P=4 \pi r^{2} n_{i} t^{0 i} \sim \frac{4 G \omega^{2} \mu^{4} \sin ^{4} \chi}{225 c^{5} R^{6}}$.

If, in agreement with Eqs. (13)-(15), we put $B \sim 2 \mu / R^{3}$, the above expression for $P$ becomes

$P \sim \frac{G \omega^{2} B^{4} R^{6} \sin ^{4} \chi}{(30)^{2} c^{5}}$

Let us compare this power with the power, $P_{q}$, radiated for a rotating star of mass $m$, radius $R$, pulsation $\omega$, and equatorial eccentricity $e$, in the quadrupole approximation. From Weinberg (1972), for example,

$P_{q} \sim \frac{32 G \omega^{6} m^{2} R^{4} e^{2}}{5 c^{5}}$

So, assuming $\chi \approx \pi / 2$, we have

$\frac{P_{q}}{P} \sim 5 \times 10^{3} \frac{\omega^{4} m^{2}}{R^{2} B^{4}} e^{2}$.

This relation says that, given $e$, the lower $\omega$ is and the higher $R$ is, the better the ratio in favor of the gravitational radiation directly generated by the e.m. field of the star.

For $\omega \sim 2 \pi \times 10^{2} \mathrm{~s}^{-1}, m \sim 2 \times 10^{33} \mathrm{~g}, R \sim 10 \mathrm{~km}$, $B \sim 10^{15} \mathrm{G}$, we get $P_{q} / P \sim 3 \times 10^{9} e^{2}$. That is to say: for $e<10^{-5}, P$ is higher than $P_{q}$, but, as it is seen from Eq. (49), the relation $P_{q} / P$ depends dramatically on $\omega$. Then, for lower values of $\omega$, all other parameters being equal, $P$ would dominate $P_{q}$ largely for any reasonable value of $e$. On the other hand, for the above parameters, $B$ could induce an eccentricity, $e$, of that order, $e \sim 10^{-5}$, or perhaps more, depending on the value of a certain "distortion parameter", $\beta$. See Bonazzola \& Gourgoulhon (1996), Bocquet et al. (1995), and Palomba (2001), specially Eq. (3) in the last reference.

To finish this discussion, let us put the amplitude of the gravitational wave given by Eq. (42) as a function of $B \sim$ $2 \mu / R^{3}$. We obtain

$h \sim \frac{G B^{2} R^{3} \sin ^{2} \chi}{30 c^{4} r}$

As we have reported before (Rankin 1990), $\chi \sim \pi / 2$ is one of the poles of the histogram of the observational values of $\chi$. So, let us place ourselves in this most favorable case and put $\sin \chi \sim 1$ in the above expression of $h$. Then for an hypothetical magnetar of $R \sim 10 \mathrm{~km}, B \sim 10^{15} \mathrm{G}$, at a distance of $\sim 3 \mathrm{kpc}$, we find $h \sim 2 \times 10^{-25}$. Now, the future Euro-third generation gravitational wave antenna (see http://www.astro.cf.ac.uk/ geo/euro) will attain a level of detection of $h \sim 10^{-23}$, operating at a frequency of $20 \mathrm{~Hz}$. But, according to Palomba (2001), the spin-down time of a magnetar to a final frequency of $10 \mathrm{~Hz}$, is about five days, for $B=10^{15} \mathrm{G}$. So, considering an integrating observation time of $\sim$ one day, the above amplitude of $h=2 \times 10^{-25}$, corresponding to a hypothetical magnetar in our galaxy, could be detected. Since $h$ in Eq. (50) goes as $B^{2}$, the observational situation could be better for new born magnetars, where $B$ would be larger, although, in this case, the integrating observational times would be shorter. Also, the frequencies of the gravitational wave emitted by the magnetar would be higher than the above $10 \mathrm{~Hz}$ at the end of the spin down time. So, gravitational wave detectors, such VIRGO and LIGO could be used in this case.

As far as we know, the most prominent candidate for this detection is the soft gamma ray repeater SGR $1900+14$, in the Galactic plane, recently considered by Ioka (2001). This author suggests a value slightly greater than $10^{14} \mathrm{G}$ for the magnetic field at the surface of the object, while Palomba (2001) reports a value of $(5.1-8.6) \times 10^{14} \mathrm{G}$. These values are slightly smaller than the $10^{15} \mathrm{G}$ considered in the above estimation. Unfortunately, this object is at a distance of $14 \mathrm{kpc}$ (Hurley et al. 1996), which is greater than the distance of $3 \mathrm{kpc}$ used in the estimation, that again reduces the possibility of attaining the level of detection.

Concerning the strongly magnetized white dwarfs given by Heyl (2000), using Eq. (50) for $\chi \sim \pi / 2$, one sees that, in this case, the quadrupole gravitational radiation dominates over the one calculated in the present paper.

\section{Appendix A}

Let us prove that the integral

$I_{L 1}=\int_{R}^{r} \frac{j_{L}\left(2 \omega r^{\prime}\right) \mathrm{e}^{2 i \omega r^{\prime}}}{r^{\prime}} \mathrm{d} r^{\prime}$

is order $\alpha(\omega R)^{L}+\beta(\omega R)^{3} \ln (\omega R)$. 
One can find in any handbook on Bessel functions that

$j_{L}(z)=z^{L} \sum_{k=0}^{\infty} \frac{(-1)^{k}}{k !(2 L+2 k+1) ! ! 2^{k}} z^{2 k}$

Then, since

$\mathrm{e}^{i z}=\sum_{l=0}^{\infty} \frac{(i z)^{l}}{l !}$

we will have

$j_{L}(z) \mathrm{e}^{i z}=z^{L} \sum_{k=0}^{\infty} \sum_{l=0}^{\infty} \frac{(-1)^{k} i^{l}}{2^{k} k !(2 L+2 k+1) ! ! l !} z^{2 k+l}$.

On the other hand, we can write

$I_{L 1}=(2 \omega)^{3} \int_{2 \omega R}^{2 \omega r} \frac{j_{L}(z) \mathrm{e}^{i z}}{z^{4}} \mathrm{~d} z$.

Then, substituting Eq. (A.4) in this integral, we get

$$
\begin{aligned}
I_{L 1}= & (2 \omega)^{3} \\
& \times\left\{\sum_{k, l=0}^{\infty} \frac{(-1)^{k} i^{l} z^{2 k+l+L-3}}{2^{k} k !(2 L+2 k+1) ! ! l !(2 k+l+L-3)}\right. \\
& \left.+\sum_{k, l}^{\prime \prime} \frac{(-1)^{k} i^{l}}{2^{k} k !(2 L+2 k+1) ! ! l !} \ln z\right\}_{2 \omega R}^{2 \omega r} .
\end{aligned}
$$

Where $\Sigma^{\prime}\left(\Sigma^{\prime \prime}\right)$ denotes the summation for values of $k$, and $l$ verifying $2 k+l+L-3 \neq 0(2 k+l+L-3=0)$.

But the integrand in Eq. (A.5) goes, for high values of $z$, as $1 / z^{5}$. This means that, since $\omega r$ is extremely high, the contribution to the integral from the upper limit, in a very good approximation, is negligible, and, according to Eq. (A.6), becomes

$$
\begin{aligned}
I_{L 1}= & -(2 \omega)^{3} \\
& \times\left\{\sum_{k, l=0}^{\infty} \frac{(-1)^{k} i^{l} z^{2 k+l+L-3}}{2^{k} k !(2 L+2 k+1) ! ! l !(2 k+l+L-3)}\right. \\
& \left.\times \sum_{k, l}^{\prime \prime} \frac{(-1)^{k} i^{l}}{2^{k} k !(2 L+2 k+1) ! ! l !} \ln z\right\}
\end{aligned}
$$

that is to say

$$
\begin{aligned}
I_{L 1}= & -\frac{1}{R^{3}} \\
& \times\left\{(\omega R)^{L} \sum_{k, l=0}^{\infty} \frac{8(-1)^{k} i^{l}(\omega R)^{2 k+l}}{2^{k} k !(2 L+2 k+1) ! ! l !(2 k+l+L-3)}\right. \\
& \left.+(\omega R)^{3} \ln (\omega R) \sum_{k, l}^{\prime \prime} \frac{8(-1)^{k} i^{l}}{2^{k} k !(2 L+2 k+1) ! ! l !}\right\}
\end{aligned}
$$

which shows that $I_{L 1}$ goes as $\alpha(\omega R)^{L}+\beta(\omega R)^{3} \ln (\omega R)$, as we wanted to prove.

Acknowledgements. We want to thank Chus Martin, J.A. Pons and M. Portilla for some valuable suggestions. We also acknowledge the anonymous referee for useful suggestions and comments.

This work has been supported by Spanish MCyT (grants AYA 2000-2045 and AYA 2001-3490-C02-02) and the EU Program "Improving the Human Research Potential and Socio-Economic Knowledge Base" (Research Training Network contract HPRN-CT-2000-00137)

\section{References}

Bocquet, M., Bonazzola, S., Gourgoulhon, E., \& Novak, J. 1995, A\&A, 301, 757

Bonazzola, S., \& Gourgoulhon, E. 1996, A\&A, 312, 675

Deutsch, A. J. 1955, Ann. Astrophys., 18, 1

Heyl, J. S. 2000, MNRAS, 317, 310

Hurley, K., Li, P., Vrba, F., et al. 1996, ApJ, 463, L13

Ioka, K. 2001, MNRAS, 327, 639

Landau, L., \& Lifshitz, E. 1962, The Classical Theory of Fields (Addison-Wesley, Reading, Mass)

Palomba, C. 2001, A\&A, 367, 525

Rankin, J. M. 1990, ApJ, 352, 247

Rezzolla, L., Ahmedov, B. J., \& Miller, J. M. 2000, MNRAS, 1, 19

Weinberg, S. 1972, Gravitation and Cosmology (John Wiley \& Sons) 